USITP-93-11

May 1993

\title{
ANYONS ON HIGHER GENUS SURFACES - a constructive approach
}

\author{
T. H. Hansson ${ }^{\dagger}$, Anders Karlhede ${ }^{\dagger}$ and Erik Westerberg \\ hansson@vana.physto.se ak@vana.physto.se wes@vana.physto.se \\ Department of Physics \\ University of Stockholm \\ Box 6730 \\ S-113 85 Stockholm \\ Sweden
}

\begin{abstract}
We reconsider the problem of anyons on higher genus surfaces by embedding them in three dimensional space. From a concrete realization based on three dimensional flux tubes bound to charges moving on the surface, we explicitly derive all the representations of the spinning braid group. The component structure of the wave functions arises from winding the flux tubes around the handles. We also argue that the anyons in our construction must fulfil the generalized spin-statistics relation.
\end{abstract}

PACS number: 03.65.-w, 03.65.Bz

${ }^{\dagger}$ Supported by the Swedish Natural Science Research Council 
Anyons, i.e. particles with fractional statistics can exist in two space dimensions [1]. The statistical angle, $\theta$, which by definition is the phase acquired by the wave function under the clock-wise interchange of two particles, is an arbitrary real number, defined mod $2 \pi$, if the particles move on an infinite plane. When the particles move on surfaces with more complicated topology, $\theta / \pi$ is restricted to special rational values. On the sphere, an infinitesimal loop traced by one of the particles can also be thought of as a big loop enclosing all the other particles. This implies a relation between $\theta$, the number of particles and the spin of the particles. On higher genus surfaces the situation is more complicated and was clarified only when Einarsson realized that the relevant representations of the braid group on the torus are multi-dimensional, and the wave functions hence have several components [2]. A fair amount of work concerning anyons on the torus exists, in particular it has been shown how to recover the component structure of the wave functions in the Chern-Simons (CS) approach [3, 4, 5]. Imbo and March-Russel [6] and Einarsson[7] generalized the results for the torus to surfaces of arbritrary genus. The relations between the number of particles, $N$, the dimensionality of the representation, $d$, (i.e. the number of components of the wave function) and the statistics, $\theta / \pi=p / q$, depend on the spin of the anyons [8, 9, 10]. This is because the coupling of the spin to the curvature of the surface gives a phase change in the wave function when the particle moves. Einarsson pointed out that to get agreement with the results from Chern-Simons theory, one must assume that the anyons carry a spin given by the generalized spin-statistics relation $s=\theta / 2 \pi$. This is consistent with the explicit calculations of spin in CS theories [11] and Maxwell-CS theories [12, 13]. The relations pertinent to anyons with spin $s=\theta / 2 \pi$ on an orientable genus $g$ surface, derived in refs. [6] and [[], read

$$
\theta / \pi=M / N=p / q, \quad d=q^{g} \quad,
$$

where $M$ is an arbitrary integer and $p$ and $q$ are relative prime (defined by $p / q=M / N)$. Thus for a given number of anyons on a given surface, the only freedom is the integer $M$, which uniquely determines the statistics and the number of components of the wave function.

The possible values for the statistical angle $\theta$ in (1) can be obtained as follows, see e.g. [14]. Embed the surface with $N$ bosons in three dimensions, and attach a three dimensional tube of magnetic flux $2 \theta$ to each particlef. One such tube crosses the surface at the position of each particle, and there is no magnetic flux flowing through the surface anywhere else, see Fig. 1. By the

\footnotetext{
${ }^{1}$ We put $c=\hbar=e=1$ so that a unit quantum of flux is $2 \pi$.
} 
Aharonov-Bohm effect, this flux turns the particles into anyons of statistics $\theta$. By Dirac's quantization condition, they must originate in an integer number, $M$ say, of magnetic monopoles inside the surface. The flux through each particle is therefore $2 \theta=2 \pi M / N=2 \pi p / q$.

In this letter, we derive all representation of spinning anyons from this three dimensional realization. The component structure of the wave functions, as well as the explicit representations for the generators of the braid group, are derived from simple physical properties of the three dimensional realization. For simplicity we imagine that the flux tubes end in $M$ anti-monopoles outside the torus and that all $2 M$ monopoles are kept fixed in space. The dynamics of the fluxes and monopoles will not be relevant for our arguments. The details of how the flux tubes are attached to the particles are not important, but below we will comment on how this can be achieved via a constraint in the Hamiltonian.

The principle behind our construction is that a quantum state of the anyons is specified not only by the particle positions, but also by the values of noncontractible Wilson loops, $e^{i \oint A}$, on the surface. We give the details of the construction for the torus; the generalization to higher genus surfaces will be obvious. Let $\rho_{i}$ be an operator that moves particle $i$ around the loop $\rho_{i}$, see Fig. 1. We assume that the eigenvalues of the quantum mechanical operator $\rho_{i}$ are the possible values the Wilson loop measuring the (three dimensional) flux through $\rho_{i}$. The operator $\tau_{j}$ is defined analogously. Classically, when an anyon moves on the torus, a three dimensional flux tube is dragged along. Moving the anyon once around $\rho_{i}$ restores the particle configuration but wraps a tube of $p / q$ flux quanta around inside the torus, thus changing the flux through $\tau_{j}$. Since we have assumed that the eigenvalues of the operators are the Wilson loops, it follows that the quantum mechanical operators $\rho_{i}$ and $\tau_{j}$ do not commute. Consider an eigenstate, $\left|k ; \vec{x}_{i}\right\rangle_{\tau}$, of the operator $\tau_{1}$. Acting on this state with $\rho_{1}$, gives a new state, $\left|k+1 ; \vec{x}_{i}\right\rangle_{\tau}$, since the flux through $\tau_{1}$ has changed. Repeated actions of $\rho_{1}$ gives new states until, after $q$ steps, an integer number of flux quanta, $p$, have been added. The initial state has then been retrieved, possibly up to a phase: $\rho_{1}^{q}\left|k ; \vec{x}_{i}\right\rangle_{\tau}=e^{i q \eta}\left|k ; \vec{x}_{i}\right\rangle_{\tau}$. Choosing the obvious phase convention we have,

$$
\rho_{1}\left|k ; \vec{x}_{i}\right\rangle_{\tau}=e^{i \eta}\left|k+1 ; \vec{x}_{i}\right\rangle_{\tau} \quad, k=0,2, \ldots q-1 \quad \text { and } \quad\left|q ; \vec{x}_{i}\right\rangle_{\tau}=\left|0 ; \vec{x}_{i}\right\rangle_{\tau}
$$

The phase $e^{i \eta}$ corresponds to a constant flux flowing through the hole of the torus. The $q$ states are the $q$ components of the wave function on the torus. Taking the $j^{\text {th }}$ particle around the loop $\rho_{j}$ will have the same effect as $\rho_{1}$ except that the enclosed flux will also get a contribution from the enclosed 
$(j-1)$ flux tubes connected to the particles. Keeping track of signs we get $\rho_{j}=e^{-2 i \theta(j-1)} \rho_{1}$. The eigenstates to the $\rho$ 's are the superpositions

$$
\left|l ; \vec{x}_{i}\right\rangle_{\rho}=\frac{1}{\sqrt{q}} \sum_{k} e^{-2 i k l \theta}\left|k ; \vec{x}_{i}\right\rangle_{\tau} \quad, \quad l=0,1, \ldots q-1
$$

with eigenvalues $e^{i \eta} e^{2 i l \theta}$ measuring the enclosed flux.

Next consider the action of the generator $\tau_{1}$. In analogy to the $\rho$ 's, the $\tau$ 's decrease the flux as measured by the $\rho$ 's by $2 \theta$ :

$$
\tau_{1}\left|l ; \vec{x}_{i}\right\rangle_{\rho}=e^{i \gamma}\left|l-1 ; \vec{x}_{i}\right\rangle_{\rho}
$$

where the phase $e^{i \gamma}$ stems from a constant flux flowing inside the torus. From Fig. 1 it is also clear that $\tau_{j}=e^{2 i \theta(j-1)} \tau_{1}$ since the enclosed flux decreases by $2 \theta(j-1)$ due to the encircled $(j-1)$ flux tubes. The eigenstates of $\tau_{j}$ are the states $\left|k ; \vec{x}_{i}\right\rangle_{\tau}$, with eigenvalues $e^{i \gamma} e^{2 i k \theta}$. As promised, the eigenvalue measures the flux through $\tau_{1}$ since $k 2 \theta$ is the flux added when acting with $\left(\rho_{j}\right)^{k}$, and $\gamma$ is the "external" flux. (The picture in Fig.1 is classical; it does not correspond to a $\tau$ (or $\rho$ ) eigenstate.)

Finally, we note that the local interchange $\sigma_{i}$ of two particles does not change the component structure but only causes the wave function to change with the familiar statistical phase $e^{i \theta}$ so that

$$
\sigma_{i}\left|k ; \vec{x}_{i}\right\rangle_{\tau}=e^{i \theta}\left|k ; \vec{x}_{i}\right\rangle_{\tau} \quad \text { and } \quad \sigma_{i}\left|l ; \vec{x}_{i}\right\rangle_{\rho}=e^{i \theta}\left|l ; \vec{x}_{i}\right\rangle_{\rho}
$$

The representation of $\tau_{j}, \rho_{j}$ and $\sigma_{j}$ in either the $\left\{\left|k ; \vec{x}_{i}\right\rangle_{\rho}\right\}$ or the $\left\{\left|l ; \vec{x}_{i}\right\rangle_{\tau}\right\}$ basis reproduces exactly the braid group representations found by Einarsson for anyons on the torus [2].

We can now proceed to construct a path integral representation for the propagator, or partition function, for the anyon system using the conventional recipe: 1. Divide the paths according to homotopy classes; 2. Perform the sum over paths in each class separately; 3. Multiply each contribution with an appropriate phase-factor and sum over all classes. Note that for a path to connect the states $\left|k ; \vec{x}_{i}\right\rangle$ and $\left|k^{\prime} ; \vec{x}_{i}^{\prime}\right\rangle$, the particles must wind altogether $k-k^{\prime}$ modulo $q$ turns in the $\rho$-direction. Also, when calculating the relative phases appropriate to the different homotopy classes, one must consistently use one representation for the braid group generators $\tau_{j}, \rho_{j}$ and $\sigma_{j}$.

The generalization to higher genus surfaces is trivially obtained by representing the genus $g$ surface as a sphere with $g$ handles. The statistics is independent of genus since $M$ monopoles inside give $M / N=p / q$ flux quanta per particle and hence statistics $\theta / \pi=p / q$. Particle transport around different 
handles commute (since winding around handle A does not affect the Wilson loops around handle B) while the generators on the same handle behave as on the torus. There are hence $q$ inequivalent windings on each handle and all in all $q^{g}$ components of the wave function and the relations (1) are recovered.

Three comments are in order. First, the number of components, for the torus, is $q$ and not $q^{2}$ which might naively have been guessed since we can wind flux tubes around both holes of the torus. This is because the component structure is not caused by the total flux through the hole but by the flux added when acting with $\rho_{i}$ or $\tau_{i}$ (i.e. when moving one particle around). Since $\rho$ and $\tau$ do not commute, only one of them will generate components. If we diagonalize $\rho$, then $\tau$ generates $q$ components and vice versa. Second, although the three dimensional embedding distinguishes the two holes in the torus, the two dimensional physics is symmetric; which hole one adds flux through just corresponds to a choice of basis. Third, we reproduced the relations corresponding to anyons with fractional spin in accordance with the generalized spin-statistics relation. Although our understanding of this is not complete, we shall argue below that this is no coincidence.

We stressed that the details of how flux is bound to the charges is of no relevance for the argument about the component structure. From that point of view we could stop here. It is, however, interesting to ask whether the flux tube picture can be made dynamical, and without going into any details we will argue two points: 1. Any consistent dynamical scheme will imply phases corresponding to particle transport in accordance with eqs. (2), (4) and (5). 2. Any flux tube-charge composite will carry fractional spin in accordance with the generalized spin-statistics relation.

One way to bind flux to charge on the surface is to use a constraint as in ref. [15]. On a plane it is well understood how this construction works: Describe the $N$ charged particles in the xy-plane by a path-integral and couple them minimally to the gaugepotential $\vec{a}=\left(A_{x}, A_{y}\right)$ which is the restriction of the 3-d vectorpotential $\vec{A}$, which describes the flux tubes, to the xy-plane. Next, tie the charges to the flux tubes by introducing a delta-functional via the identity $1=\int \mathcal{D} b \delta\left[\left(b(\vec{x})-2 \theta \sum_{i=1}^{N} \delta^{2}\left(\vec{x}_{i}\right)\right]\right.$ in the path integral description of the system. This constraint is then exponentiated using a Lagrange multiplier field $a_{0}$, and as shown in [15, the resulting $a_{0} b$ term (where $b=B_{z}$ ) in the action is nothing but a Chern-Simons (CS) term in Coulomb gauge.

On the torus, everything works locally as on the plane. After exponentiating the constraint one has a path integral over $b$ and $a_{0}$ and $a_{0} b$ in the action. However, this is not equivalent to the full CS action on the torus; the non-trivial Wilson loops are missing since we integrate over $b$ only. When 
quantizing the full CS action, i.e. integrating over all gauge fields $a_{\mu}$, then the two non-trivial Wilson loops, corresponding to $\rho$ and $\tau$, are conjugate variables and give a single quantum degree of freedom [16, 5]. The component structure explained above exactly corresponds to a single quantum degree of freedom with the same spectrum as the Wilson loop mode, and we believe that the two descriptions are indeed equivalent. Our construction then, is a "mixed" one: The statistical phase is implemented by flux tubes (or gauge fields), and the wave functions are single valued but have several components associated with $\rho$ and $\tau$. In the description of anyons with a full CS term [5], the Wilson loop mode replaces the component structure.

As discussed in [12] and [17, the composites are anyons with statistical angle $\theta$. Note that this is not self evident. Naively one would expect a phase $2 \theta$ since there are equally big contributions from the charge moving relative to the flux, and the flux relative to the charge.2 However, there is a contribution to the phase from the CS term, i.e. from the constraint, that cancels half of the naive contribution. Since $a_{i}$ is the restriction of $A_{i}$ to the surface, this proves the assertion that the wave-functions pick up phases equal to the Aharonov-Bohm phase corresponding to a unit test charge following the same trajectory. In particular this means that the statistical phase corresponding to interchanging two particles will be $\theta$.

The simplest way to see that anyons in the CS theory carry spin is to calculate the corresponding spinfactors, as explained in refs. [19], 20 and [21]. Another way is to directly evaluate the canonical angular momentum from the Lagrangian. Both methods give results in accordance with the spinstatistics connection. A A third way to understand the spin is by appealing to the topological proof of the spin-statistics theorem given by Balachandran et. al. [9, 10. This proof does not require Lorentz invariance, but is based on the following assumptions: i) A continuous, spacelike, "frame" can be associated with each point on the worldline of a particle. ii) There exist antiparticles that carry mirror-reflected frames. iii) Particle-antiparticle pairs can be created and annihilated only if their frames are aligned (see Fig. 2b). In our flux tube picture there is a very natural way to attach frames to the anyons. Define $\hat{e}_{1}$ to be the charge of the particle times the projection of the unit tangent vector to the magnetic flux tube at the position of the anyon $\left(\hat{e}_{1}\right.$ normalized to unit length) and $\hat{e}_{2}$ to be the (normalized) cross-product between the tangent vector and $\hat{e}_{1}$ (Fig. 2a). It is clear that the frame of an anti-anyon will be the mirror

\footnotetext{
${ }^{2}$ Such composites were named "cyons" by Goldhaber 18 .

3 The calculations in [13] show that it is not crucial that the anyons are point like, and it is demonstrated that the spin-statistics connection holds for a large class of Lagrangians.
} 
image of the frame of an anyon and also that the frames are aligned as required in any annihilation/creation process (Fig. 2b). There is a difficulty in that the frame is not well defined if the flux tube is perpendicular to the surface, but if we modify the construction and exclude this possibility then the flux tube picture gives a very nice concrete realization of the assumptions going into the topological proof of the spin-statistics theorem.

Again we must generalize the treatment to a curved space. This we have not done in general, but we have checked that in the case of a sphere the so called non-covariant, or twist, form of the spinfactor 19, 21 picks up precisely that dependence on the curvature that is necessary for consistency with the braid group analysis of Einarsson. Also, it seems to us that the argument based on the topological proof of the spin-statistics theorem generalizes directly to a curved surface.

Acknowledgement: We thank Ansar Fayyazuddin for many helpful discussions that forced us to sharpen our arguments. We also thank Martin Roček for discussions.

\section{References}

[1] J. M. Leinaas, and J. Myrheim, Nuovo Cimento, 37B (1977)1.

[2] T. Einarsson, Phys. Rev. Lett., 64 (1990)1995.

[3] R. Iengo and K. Lechner, Nucl. Phys., B346 (1990)551.

[4] K. Lechner, Anyon Physics on the Torus, Thesis, 1991.

[5] A. Fayyazuddin, On the origin of multi-component anyon wave functions, Preprint, Stockholm University, USITP-92-15, Nucl. Phys. B, in press, 1992.

[6] T. D. Imbo and J. March-Russel, Phys. Lett., B 252 (1990)84.

[7] T. Einarsson, Mod. Phys. Lett., B5 (1991)675.

[8] F. Wilczek, Phys. Rev. Lett., 49 (1982)957.

[9] A.P. Balachandran, A. Daughton, Z.-C. Gu, G. Marmo, R. D. Sorkin, and A. M. Srivastava, Mod. Phys. Lett., A5 (1990)1575. 
[10] A.P. Balachandran, A. Daughton, Z.-C. Gu, G. Marmo, R. D. Sorkin, and A. M. Srivastava, Spin-Statistics theorems with no relativity or field theory, Preprint, Syracuse University Preprint SU-4228-433, May 1990.

[11] C. R. Hagen, Phys. Rev., D31 (1985)2135.

[12] T. H. Hansson, M. Roček, I. Zahed, and S. C. Zhang, Phys. Lett., B214 (1988) 475 .

[13] A. Karlhede S. Artz, T. H. Hansson and T. Staab, Phys. Lett., B 267 (1991)389.

[14] T. Einarsson, Anyons and Antiferromagnets:Two Two-dimensional Topics, Thesis, Chalmers University of Technology, Göteborg 1992.

[15] S-C. Zhang, T. H. Hansson and S. Kivelson, Phys. Rev. Lett., 62 (1989)82.

[16] A. P. Polychronakos, Ann. Phys., 203 (1990)231.

[17] A. S. Goldhaber and R. Mackenzie, Phys. Lett., B214 (1988)471.

[18] A. S. Goldhaber, Phys. Rev. Lett., 49 (1982)905.

[19] A. M. Polyakov, Mod. Phys. Lett., 3 (1988)325.

[20] J. Grundberg, T.H. Hansson, A. Karlhede, and U. Lindström, Phys. Lett., B218 (1989)321.

[21] J. Grundberg, T.H. Hansson, and A. Karlhede,, Nucl. Phys., B347 (1990)420. 